Issued by Sandia National Laboratories, operated for the United States Department of Energy by Sandia Corporation.

NOTICE: This report was prepared as an account of work sponsored by an agency of the United States Government. Neither the United States Government, nor any agency thereof, nor any of their employees, nor any of their contractors, subcontractors, or their employees, make any warranty, express or implied, or assume any legal liability or responsibility for the accuracy, completeness, or usefulness of any information, apparatus, product, or process disclosed, or represent that its use would not infringe privately owned rights. Reference herein to any specific commercial product, process, or service by trade name, trademark, manufacturer, or otherwise, does not necessarily constitute or imply its endorsement, recommendation, or favoring by the United States Government, any agency thereof, or any of their contractors or subcontractors. The views and opinions expressed herein do not necessarily state or reflect those of the United States Government, any agency thereof, or any of their contractors.

Printed in the United States of America. This report has been reproduced directly from the best available copy.

Available to DOE and DOE contractors from

Office of Scientific and Technical Information

P.O. Box 62

Oak Ridge, TN 37831

Prices available from (703) 605-6000

Web site: http://www.ntis.gov/ordering.htm

Available to the public from

National Technical Information Service

U.S. Department of Commerce

5285 Port Royal Rd

Springfield, VA 22161

NTIS price codes

Printed copy: A03

Microfiche copy: A01

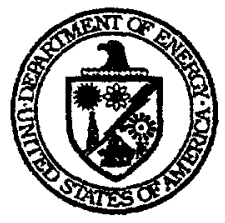




\section{DISCLAIMER}

Portions of this document may be illegible in electronic image products. Images are produced from the best available original document. 
SAND 2000-8218

Unlimited Release

Printed March 2000

\title{
Electrokinetic Pumps and Actuators
}

\author{
Phillip H. Paul \\ MicroTechnologies Department, 8355 \\ Sandia National Laboratories \\ Livermore CA 94550
}

\begin{abstract}
Flow and ionic transport in porous media are central to electrokinetic pumping as well as to a host of other microfluidic devices. Electrokinitic pumping provides the ability to create high pressures (to over $10,000 \mathrm{psi}$ ) and high flowrates (over $1 \mathrm{~mL} / \mathrm{min}$ ) with a device having no moving parts and all liquid seals. The electrokinetic pump (EKP) is ideally suited for applications ranging from a high pressure integrated pump for chip-scale HPLC to a high flowrate integrated pump for forced liquid convection cooling of high-power electronics. Relations for flowrate and current fluxes in porous media are derived that provide a basis for analysis of complex microfluidic systems as well as for optimization of electrokinetic pumps.
\end{abstract}


Acknowledgement The author gratefully acknowledges Dr. Don Arnold, Dr. Dave Rakestraw, Dr. Greg Frye-Mason, Mr. Ken Hencken and Mr. Mike Oborny for their contributions to and participation in this project, and Dr. Tim Shepodd for supplying samples of porous polymers. The author also acknowledges Dr. Kate Smith, Dr. Dave Neyer, Dr. Susanne Wallenberg, Dr. Cris Bailey, Dr. Stu Griffiths, Dr. Bob Nilson, Dr. Charlie Hasselbrink and Dr. Jason Rehm for many helpful discussions. The author acknowledges the support and assistance of Dr. Jill Hruby, who managed this project. This work was supported by Sandia National Laboratories, Laboratory Directed Research and Development. 


\title{
Electrokinetic Pumps and Actuators
}

\author{
Phillip H. Paul \\ MicroTechnologies Department, 8355 \\ Sandia National Laboratories \\ Livermore CA 94550
}

Introduction Flow and ionic transport in porous media is central to electrokinetic pumping as well as to a host of other microfluidic devices. Electrokinitic pumping provides the ability to create high pressures (to over $10,000 \mathrm{psi}$ ) and high flowrates (over $1 \mathrm{~mL} / \mathrm{min}$ ) with a device having no moving parts and all liquid seals. The electrokinetic pump (EKP) is ideally suited for applications ranging from a high pressure integrated pump for chip-scale HPLC to a high flowrate integrated pump for forced liquid convection cooling of high-power electronics. Here relations for flowrate and current fluxes in porous media are developed that provide the basis for analysis of complex microfluidic systems as well as for optimization of electrokinetic pumps.

In previous studies [1] relationships were given describing electrokinetic pumps in the limit of constant properties. Subsequent experiments have shown that axial non-uniformities can play a significant role in the performance of EKP's and other microfluid devices. Consideration of the observed effects has driven the development of a more complete model of flow in porous media that explains much of the experimental data and suggests several methods to achieve substantial improvements in EKP performance.

Flux Relations Consider a plug or matrix of homogeneous non-conducting porous material of thickness $L$, cross sectional area $A$, and a fractional open volume $\phi$ (the porosity) that is saturated with a fluid having viscosity $\mu$ and electrical permittivity $\varepsilon$ both taken as constant (see appendix B), and electrical conductivity $\sigma_{f}$. An electrical potential $\Delta \mathrm{V}$ and a pressure difference $\Delta P$ are externally applied across the plug. A layer of immobile electrolyte ions is taken to be absorbed onto the material surface forming the so-called Stern layer. Any net charge in this layer must be balanced by an excess of net charge, as mobile ions of opposite sign, in the adjacent fluid layer, the so-called diffuse layer. The Stern and diffuse layers together represent the so-called double layer. The surface separating these two layers is taken as the plane-of-shear. In a continuum description, the solid surface is then represented by a uniform surface charge density and a no-slip boundary condition.

The double layer is characterized by a thickness $\lambda_{\mathrm{DL}}$ (the integral thickness of the double layer potential field as normalized by the zeta potential) which is in general less than or equal to the Debye length, $\lambda_{d}$, in the bulk electrolyte. Here the Debye length is taken in terms of the ionic strength of the electrolyte. To develop a continuum description of the system requires three 
assumptions: The radius of curvature of features on the solid matrix are large compared to the double layer thickness, in essence the double layer is 'flat;' the double layer thickness is large compared to the surface site-to-site spacing, in essence the double layer is 'smooth,' and; the double layer is of finite thickness compared to the pore diameter. On silica the charge site surface density is order 0.6 sites $/ \mathrm{nm}^{2}$. The smooth assumption is taken as valid is the double layer thickness is greater than $1 / 2$ the average site-to-site spacing, requiring ionic strengths of less than $0.2 \mathrm{~mol} / \mathrm{L}$. In the following it will be shown that the model developed is suitable for pore diameters greater than of order ten Debye lengths.

The flow through the plug is taken in the limit of negligible inertial effects, a condition termed 'creeping' or Stoke's flow. Current transport is taken in the limit of negligible magnetic interaction and negligible solid conductivity, and is thus due to ionic conduction and charge convection (e.g. the transport of net charge in the double layer by fluid convection). The effect of charge 'hopping' along the surface is neglected. The plug is taken to be large compared to the pore/grain size and considered to contain a statistically representative sample of the porous (solid) matrix. The plug is also taken to be small compared to the scale of axial (flow direction) gradients in the driving fields and in property variations. In these limits the governing equations can be one-dimensionalized along the flow direction by formally volume averaging the equations over the plug. Typically area integrals are employed to define fluxes. However in a porous medium, volume integrals are required to define fluxes in terms of apparent axial gradients in the driving fields.

The electrical power dissipation in the plug is given by

$$
\text { Pow }=\int_{C P} \vec{J} \bullet \vec{E} d v
$$

where this integral is over the connected pore volume within the plug., $J$ and $E$ are the current flux and electric fields in the fluid within the plug. For negligible zeta potential (e.g. at the material isoelectric point, IEP) the current flux is given by $\vec{J}=\sigma_{f} \vec{E}$. Equating these to an Ohmic power dissipation, in terms of an effective conductivity (i.e. $P o w=I^{2} L / A \sigma_{\text {eff }}$ where $I$ is the current), yields

$$
\frac{\sigma_{e f f}}{\sigma_{f}}=\left.\frac{1}{v} \int \frac{\vec{E}}{c p}\right|^{2} d v \equiv \frac{1}{F}
$$

Here $v=A L$ is the total volume of the plug and $F$ is termed the 'formation factor.' A common description of a porous medium is the tortuous pore model where the pores are represented by an intertwined bundle of tubules of length $L_{\mathrm{t}}$. The magnitude of the electric field in the tubules is then $\Delta V / L_{\mathrm{t}}$. Observing that the ratio of the integral over the connected pore volume to the total volume is the definition of the connected porosity $\phi_{\mathrm{cp}}$, the formation factor is then $F=$ $\tau^{2} / \phi_{\mathrm{cp}}$ where $\tau^{2}=\left(L_{\mathrm{t}} / L\right)^{2}$ is termed the tortuosity.

Johnson et al. [2] have suggested a characteristic length scale for porous media

$$
\Lambda \equiv 4 \int_{c p}|\vec{E}|^{2} d v / \int_{c p}|\vec{E}|^{2} d S_{w}
$$


where the integral in the denominator is over the wetted surface. Note, here this length scale is taken as a diameter rather than a radius as done by Johnson et al. For a straight right channel, this length scale is thus identical to the hydraulic diameter (e.g. 4 time the wetted cross sectional area divided by the wetted perimeter).

This length scale, $A$, is in fact a dynamical diameter that depends both on the pore size distribution and the connectivity. There is no direct relationship between the pore size distribution and the value of $\Lambda$. Consider a collection of straight constant diameter tubes of equal length, the collection having a distribution of diameters $P(D) d D$. For the tubes arranged in parallel, $\Lambda=\left\langle D^{2}\right\rangle /\langle D\rangle$ thus the flux favors the larger diameter tubes. However for the same set of tubes arranged in series, $\Lambda=\left\langle D^{-1}\right\rangle /\left\langle D^{-2}\right\rangle$ and the flux is limited by the small diameter tubes. Here $\left\langle D^{n}\right\rangle$ represent the $n^{\prime}$ th moment of the size distribution.

Following the conjecture of Katz and Thompson [3], Johnson, Koplik and Schwartz. [JKS, 4] have suggested the relation $F k_{D}=M \Lambda^{2}$ where $M$ is termed the pore geometry number and $\mathrm{k}_{\mathrm{D}}$ is the Darcy permeability. The value of $M$ has been shown near constant, both theoretically and experimentally, for a wide range of porous materials [see for example 5,6]. In the present formulation; for a capillary $M=1 / 32$ and for other porous materials may be as large as 1/16.

The traditional descriptions of porous materials (e.g. connected and total porosity, tortuosity, electric and hydraulic formation factors, Darcy permeability) are here replaced by the formation factor $F$, the dynamic length scale $\Lambda$ and the pore geometry number, $M$.

The formal volume averaging of the 3-dimentional governing equations yields a set of onedimensional equations for the current flux, $j$, and volume flowrate flux $q$ along the applied voltage and pressure gradients

$$
\begin{aligned}
& q=(\widetilde{v} \nabla V-\widetilde{k} \nabla P) / F \\
& j=(-\widetilde{\sigma} \nabla V+\widetilde{v} \nabla P) / F
\end{aligned}
$$

Here $\nabla \mathrm{V}$ and $\nabla \mathrm{P}$ are the axial gradients in the driving fields (here $\vec{E}=-\nabla V$ ), $\widetilde{\sigma}$ is an effective electroosmotic mobility, $\widetilde{k}=M \Lambda^{2} / \mu$, and $\widetilde{\sigma}$ is an effective electrical conductivity. These two relationships (equations 4 ) can be applied to define eight flow-current phenomena:

$\begin{array}{lll}\nabla \mathrm{V}=0 & \begin{array}{l}Q=-\widetilde{k} \nabla P A / F \\ \text { Darcy flow }\end{array} & \begin{array}{l}I=\widetilde{v} \nabla P A / F \\ \text { streaming current }\end{array} \\ \nabla \mathrm{P}=0 & \begin{array}{l}Q=\widetilde{v} \nabla V A / F \\ \text { electroosmotic flow }\end{array} & \begin{array}{l}I=-\widetilde{\sigma} \nabla V A / F \\ \text { ionic conduction/convection current }\end{array} \\ \mathrm{Q}=0 & \nabla P=\widetilde{v} / \widetilde{k} \nabla V & I=-\widetilde{\sigma}\left(1-\frac{\widetilde{v}^{2}}{\widetilde{k} \widetilde{\sigma}}\right) \nabla V A / F \\ & \text { electrokinetic pressure } & \text { blocked current }\end{array}$


All of these phenomena are well known with the possible exception of the 'blocked' current. This is analogous to the electroviscous effect, the apparent increase in viscosity that is observed in pressure-driven flow under open circuit conditions that yields a streaming potential that in turn drives a reverse electroosmotic flow. Whereas the blocked current represents an apparent decrease in conductivity that occurs under conditions of an applied potential and zero net flow. In this case, electroosmosis will transport fluid downstream, creating a pressure sufficient to drive an equal amount of fluid back upstream (pressure-driven flow through the centers of the pores). This pressure-driven flow will create a streaming current opposite to the current associated with the applied field leading to a reduction in the total observed current, hence the reduced or 'blocked' current.

These eight relationships form the basis of a number of different experimental methods for characterization of porous media (e.g. to measure the zeta potential). It must be noted that the formation factor plays a key role in the interpretation of such data. Thus $F$ must be measured separately as an intrinsic part of any media characterization study

Effective Transport Properties Relationships for the effective electroosmotic mobility and the effective conductivity can be developed following the model of Johnson, Koplik and Schwartz. They considered that case of current flow in a porous matrix including the condition of a finite surface conductivity on the solid. In the limit that conduction remains dominated by that in the bulk fluid they find that the effective conductivity can be written

$$
\frac{\sigma_{e f f}}{\sigma_{f}}=\left(1+\frac{4}{\Lambda} \frac{\Sigma_{S}}{\sigma_{f}}\right) \frac{1}{F}
$$

where $\Sigma_{5}$ is the surface conductivity. Revil and Glover [7] have extended this analysis to consider the excess conductivity introduced by the presence of a double layer, including the effect of variation of ionic mobilities in the double layer. A similar development is followed here with the assumption of constant mobilities for simplicity.

There are two contributions to the current flux, E-field-driven conduction and velocity-fielddriven charge convection. The later arises due to non-zero net charge density in the double layer. The Stokes equation is linear in velocity and pressure, thus the velocity and pressure fields can be linearly separated into mechanical (pressure-driven) and electrical components. The mechanical charge convection has already been separated out and appears as the pressure-gradient term in the current flux relation above. The effective conductivity then contains the contributions from E-field-driven conduction and convection. The current flux density as a function of position in the pore fluid is

$$
\vec{J}_{e}=\vec{E} \sum_{k}\left(e z_{k}\right)^{2} b_{k} N_{k}(x)+\rho_{e}(x) \vec{u}_{E}(x)
$$

Here $z$ is the charge number, $b$ is an unsigned mobility (given by $b=\eta / e$ where $\eta$ is an unsigned mobility in units of $\mathrm{cm}^{2} / \mathrm{V}-\mathrm{s}$, say), and $N$ is the concentration of the $k$ th ionic 
species (the dependence on $\mathrm{x}$ indicates a radial position-dependent field). Observing that the bulk conductivity is given by

$$
\sigma_{f}=\sum_{k}\left(e z_{k}\right)^{2} b_{k} n_{k}
$$

where $n$ is the concentration of the $k^{\prime}$ th ionic species in the bulk fluid. The current flux can be re-written as

$$
\vec{J}_{e}=\vec{E} \sigma_{f}+\vec{E} \sum_{k}\left(e z_{k}\right)^{2} b_{k}\left(N_{k}(x)-n_{k}\right)+\rho_{e}(x) \vec{u}_{E}(x)
$$

The later two terms are non-zero only in the double layer and may be cast as two types of surface conductivity: $\Sigma_{S}^{e m}$ or 'electromigration' arising from variation in ionic concentration through the double layer, and; $\Sigma_{S}^{o s}$ or 'osmotic' due to net charge convection by the electroosmotic flow. Expressions for these surface conductivites requires integration of the current flux over the double layer that first requires a solution to the Poisson-Boltzman equation.

For the plane parallel problem and for an equivalent electrolyte the solution to the PoissonBoltzman equation in the Debye-Huckel limit (i.e. $y_{k} \equiv e z_{k} \zeta / k T<2$ ) is

$$
\psi(x)=\zeta \exp \left(-x / \lambda_{d}\right)
$$

where $\mathrm{x}$ is position measured from the solid surface. It is well known that even for conditions not satisfying the Debye-Huckel limit that application of this relation as integrated across the double layer yields accurate results in relating surface charge density to the zeta potential (in fact for equivalent electrolytes the result is exact). Using this solution the surface conductivities can be written

$$
\begin{gathered}
\Sigma_{S}^{e m}=\sum_{k}\left(e z_{k}\right)^{2} b_{k} n_{k} \int_{0}^{D}\left\{\exp \left(-y_{k} \exp \left(-x / \lambda_{D}\right)\right)-1\right\} d x \\
\Sigma_{S}^{o s}=\frac{2 \varepsilon k T}{\mu} \sum_{k} n_{k} \int_{0}^{D}\left\{\exp \left(-y_{k} \exp \left(-x / \lambda_{D}\right)\right)-1\right\} d x
\end{gathered}
$$

Expanding the outer exponential in the integrand and integrating the resulting series term-byterm yields

$$
\beta \equiv \frac{\sum_{S}^{o s}+\sum_{S}^{e m}}{\sigma_{f} \lambda_{D}}=\frac{2 \sum_{k}\left(e z_{k}\right)^{2} b_{k} n_{k}\left(1+2 \varepsilon k T / b_{k}\left(e z_{k}\right)^{2}\right)\left(\exp \left(-y_{k} / 2\right)-1\right)}{\sum_{k}\left(e z_{k}\right)^{2} b_{k} n_{k}}
$$

For a 1:1 equimobile electrolyte this may be rewritten as

$$
\beta=2\left(1+\frac{2 \varepsilon k T}{\mu e^{2} b}\right)(\cosh (e \zeta / 2 k T)-1)
$$


where the two terms in the lead parenthesis represent the electromigration and osmotic contributions, respectively. For $\mathrm{KCl}$ (essentially a 1:1 equimobile electrolyte) at $300 \mathrm{~K}$, the coefficient of the osmotic term is about $1 / 2$ and therefore cannot be neglected compared to unity (the coefficient of the electromigration term).

For a 1:1 non-equimobile electrolyte the result may be written as

$$
\beta=2\left(1+\frac{4 \varepsilon k T}{\mu e^{2}\left(b_{+}+b_{-}\right)}\right)(\cosh (e \zeta / 2 k T)-1)-2 \frac{b_{+}-b_{-}}{b_{+}+b_{-}} \sinh (e \zeta / 2 k T)
$$

Depending on the sign of the zeta potential and the relative magnitudes of the mobilities of the positive and negative ions, the second term may increase or decrease the value of $\beta$. For example: the zeta potential of silica at $\mathrm{pH}$ valves above the IEP is negative thus flipping the sign of the sinh term. Keeping the zeta potential and the average of the mobilities constant hence the first term in $\beta$ constant, then $\beta\left(b_{+}>b_{-}\right)>\beta\left(b_{+}=b_{-}\right)>\beta\left(b_{+}<b_{-}\right)$. In a sodium borate buffer at $\mathrm{pH} 9$ (say) and at moderate concentrations the primary negative charge carrier is singly valent orthoborate that has a lower mobility that $\mathrm{Na}^{+}$. In a sodium phosphate buffer at $\mathrm{pH} 7.6$ (say), the primary negative charge carriers are singly and doubly valent phosphates that have higher mobilities than $\mathrm{Na}^{+}$. Thus $\beta$ ('borate' $\left.\mathrm{pH} 9\right)>\beta(\mathrm{KCl})>\beta$ ('phosphate' $\mathrm{pH} 7.5$ ).

Following the JKS result given in equation 6 , the effective conductivity may then be written

$$
\widetilde{\sigma}=\sigma_{f}\left(1+4 \frac{\lambda_{D}}{\Lambda} \beta\right)
$$

This shows that in the limit of vanishing double layer thickness as compared to the pore scale or in the limit of a vanishing zeta potential hence vanishing value of $\beta$, the effective conductivity tends to that of the bulk fluid. On the other hand for a $1: 1$ equimobile electrolyte (e.g. $\mathrm{KCl}$ ) and for silica at $\mathrm{pH} 7$ hence a zeta potential of order $60 \mathrm{mV}$, the value of $\beta$ is order 2.5. For this case and a double layer thickness order one-tenth the pore diameter the apparent conductivity may approach twice the bulk value.

The effective mobility may be written

$$
\widetilde{v}=\frac{\varepsilon \zeta}{\mu}\left(1-2 \frac{\lambda_{D}}{\Lambda}\right)^{2}
$$

This is exactly the result that is obtained from analyzing flow through a capillary and is know to be an accurate approximation for values of double layer thickness less than one-tenth the pore diameter and for values of e $\zeta / \mathrm{kT}$ less than order 5 . The correction to the classical Helmholtz-Smoluckowski mobility represents the relative velocity defect between that for infinitely thin and finite thickness double layers. Cummings et al. [8] have considered the steady incompressible Navier-Stokes equation including the product of electric field and net charge density in the double layer as a body force term. They have shown that the solution for the $3 \mathrm{D}$ velocity field of the fluid not in the double layer is given by $\vec{u}_{e t}=-(\varepsilon \zeta / \mu) \vec{E}$. The Helmholtz-Smoluckowski mobility is obtained by applying this result over the whole pore. 
For the case of a finite thickness double layer, the correction (e.g. $\tilde{v}=(\varepsilon \zeta / \mu)(1-\xi))$ is given by $\xi=\int\left(\bar{u}_{e t}-\bar{u}\right) d v / \int \vec{u}_{e t} d v$ where $u$ is the true velocity field and the integrals are over the connected pore volume.

Non-uniform Transport Properties Consider the case of a constant cross section microchannel, of length $L$ and filled end-to-end with a uniform porous matrix saturated with fluid. Potential and pressure differences are applied between the ends of the microchannel, however there are gradients in fluid composition end-to-end (possibly due to different fluid mixtures in the terminal reservoirs). The flowrate and current are conserved along the length of the system. Thus the flux equations can be combined and integrated along the length to yield

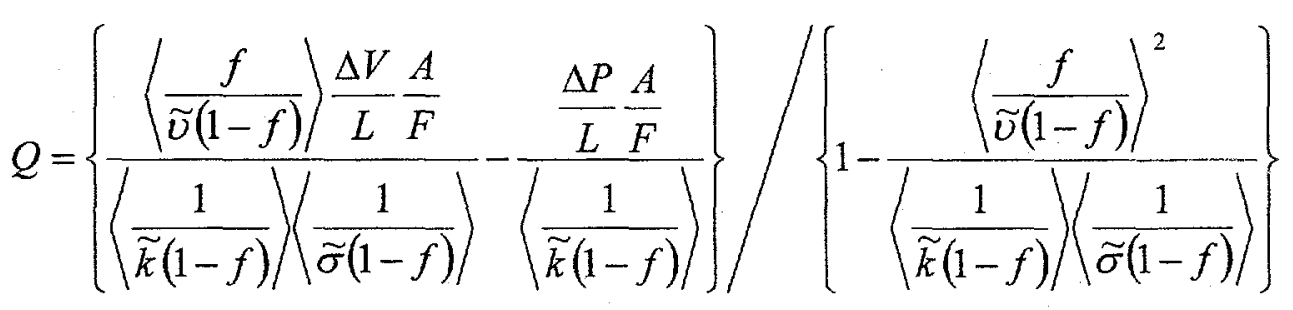

$$
I=\left\{\frac{-\frac{\Delta V}{L} \frac{A}{F}}{\left\langle\frac{1}{\tilde{\sigma}(1-f)}+\frac{\left\langle\frac{f}{\widetilde{v}(1-f)}\right\rangle \frac{\Delta P}{L} \frac{A}{F}}{\left(\frac{1}{\tilde{k}(1-f)}\right)\left(\frac{1}{\tilde{\sigma}(1-f)}\right)}\right\}} /\left\{1-\frac{\left\langle\frac{f}{\widetilde{v}(1-f)}\right)^{2}}{\left(\frac{1}{\tilde{k}(1-f)}\right)\left(\frac{1}{\tilde{\sigma}(1-f)}\right)}\right\}\right.
$$

Here $\langle-\rangle$ indicates the average of the operand over the length of the system and $f \equiv \widetilde{v}^{2} / \widetilde{k} \widetilde{\sigma}$. Here the presumption is made that the matrix properties are constant along the length (i.e. constant formation factor). In this case and for constant viscosity, the effective Darcy permeability will also be a constant. A similar formulation can be developed for the case of a set of elements connected in series where each element is described by a specific area, formation factor and transport properties. This type of analysis forms the basis for development of a microflow system design tool.

Electrokinetic Pump 'Algebra' From the table of flow phenomena above (equations 5), the electroosmotic flowrate, maximum electrokinetic pressure, and Darcy flowrate can be written

$$
\begin{gathered}
Q= \pm g\left(V_{\text {out }}-V_{\text {in }}\right) \\
\Delta P_{m}= \pm f\left(V_{\text {out }}-V_{\text {in }}\right) \\
Q=-k_{R}\left(P_{\text {out }}-P_{\text {in }}\right)
\end{gathered}
$$


respectively. Here $g=|\widetilde{v}| A / F L, f=|\widetilde{v}| / \widetilde{k}$, and $k=\widetilde{k} A / F L$. The \pm signs in these relations refer to positive (positive wall charge, e.g. alumina at $\mathrm{pH} 7$ ) and negative (negative wall charge, e.g. silica at $\mathrm{pH} 7$ ) materials.

Case I: Consider a negative electrokinetic pump (EKP) in series with a section of inactive porous material (a simple Darcy flow restrictor). The terminal inlet and outlet pressures are taken at ambient, $P_{0}$. A voltage difference $\Delta \mathrm{V}$ is applied across the EKP with the negative terminal connected at the junction between the EKP and the flow restrictor. Applying equation 18 , the pressure at the junction will be

$$
P-P_{o}=\Delta V g /\left(k_{p}+k_{R}\right)
$$

and the flowrate through the pump an thus through the flow restrictor will be

$$
Q=g \Delta V\left(1-k_{p} /\left(k_{p}+k_{R}\right)\right)
$$

Case II: Consider a positive and negative EKP connected at a junction with a Darcy flow restrictor. A voltage $\Delta \mathrm{V}_{\mathrm{A}}$ is connected across the negative EKP with the negative terminal at the junction and a separate voltage $\Delta \mathrm{V}_{\mathrm{B}}$ is connected across the positive EKP with the positive terminal at the junction. Again all terminal inlets and outlets are taken at ambient pressure. Again applying equation 18 , the pressure at the junction will be

$$
P-P_{o}=\left(g_{A} \Delta V_{A}+g_{B} \Delta V_{B}\right) /\left(k_{A}+k_{B}+k_{R}\right)
$$

The total flowrate through the restrictor will be

$$
Q=k_{R}\left(g_{A} \Delta V_{A}+g_{B} \Delta V_{B}\right) /\left(k_{A}+k_{B}+k_{R}\right)
$$

Consider the ' $\mathrm{B}$ ' pump: the flowrate is positive for $g_{B} \Delta V_{B}\left(k_{A}+k_{R}\right)>g_{A} \Delta V_{A} k_{B}$, if these quantities are equal or the inequality is reversed, the flowrate through the ' $B$ ' is zero or reversed (e.g. backflowing), respectively. A similar set of relation holds for the ' $A$ ' pump. The fraction of ' $B$ ' pump fluid is given by

$$
\frac{Q_{B}}{Q}=\frac{g_{B} \Delta V_{B}\left(k_{R}+k_{B}\right)-g_{A} \Delta V_{A}}{k_{R}\left(g_{B} \Delta V_{B}+g_{A} \Delta V_{A}\right)}
$$

A similar relation can be written for the fraction of ' $A$ ' pump fluid.

Case III: The configuration is the same as in Case II except that a single supply voltage $\Delta V$ is connected between the inlets of the two EKP's with the positive terminal at the inlet of the negative EKP (pump ' $\mathrm{A}$ '). The electrical resistance of an EKP is given by $R=F L / A \widetilde{\sigma}$. Note that depending on conditions a correction for the 'blocked' current may need to be added, but this will be taken as negligible here. Taking no current leakage through the flow restrictor and using Ohm's law to determine the voltage across each pump, the pressure at the junction is given by

$$
P-P_{o}=\frac{\Delta V}{R_{A}+R_{B}} \frac{\left(g_{A} R_{A}+g_{B} R_{B}\right)}{\left(k_{A}+k_{B}+k_{R}\right)}
$$


The flowrate through the restrictor is given by $Q=k_{R}\left(P-P_{o}\right)$.

In general it can be shown that for EKP's connected in this fashion, a pressure will be generated at the junction as long as $(\widetilde{J} / \widetilde{k} F)_{A} \neq(\widetilde{v} / \widetilde{k} F)_{B}$ a condition that may occur simply due to differences in double layer overlap between the two pumps. As a particular example consider the case where $\Lambda_{B}$ becomes of order the Debye length. In this limit both $\mathrm{k}_{B}$ and $g_{B}$ become vanishingly small (both due to significant double layer overlap) however $R_{B}$ remains finite. The junction pressure in this case is

$$
P-P_{o}=\frac{\Delta V}{R_{A}+R_{B}} \frac{g_{A} R_{A}}{\left(k_{A}+k_{R}\right)}
$$

There is negligible pressure- or electroosmotically-driven liquid flow but finite current flow through EKP ' $\mathrm{B}$ ' which thus acts as a so-called 'salt bridge'.

Electrokinetic Pump Characterization The maximum pressure generated by an electrokinetic pump (no net flowrate) is directly proportional to the voltage applied across the EKP. That is

$$
\frac{\Delta P_{m}}{\Delta V}=\frac{\widetilde{v}}{\widetilde{k}}=\frac{\varepsilon \zeta}{M \Lambda^{2}}\left(1-2 \frac{\lambda_{D}}{\Lambda}\right)^{2}
$$

For a material composed of packed, nominally monodisperse spherical beads, the KozenyCarman relation gives $k_{D}=\phi^{3} d_{\text {bead }}^{2} / 36 C(1-\phi)^{2}$ where the constant is commonly taken as $C$ $\approx 4.5$. For a bead-packed porous material, Berryman [9] has suggested the relation $F=2 / \phi(1+\phi)$ implying a tortuosity of $\tau^{2}=2 /(1+\phi)$. These give

$$
\frac{\Delta P_{m}}{\Delta V}=\frac{96 C \varepsilon \zeta}{d_{\text {bead }}^{2}}\left(1-2 \frac{\lambda_{D}}{\Lambda}\right) g(\phi)
$$

Here $g(\phi)$ is a weak function of porosity, $\varphi$, expanded about $g(\varphi=1 / 3)=1$. Equating the product of the Kozeny-Carman relation for the Darcy permeability and the Berryman relation for formation factor to $M \Lambda^{2}$ gives $\Lambda=d_{\text {bead }} / \sqrt{96 M C g(\phi)}$. For example: taking $M=1 / 32$, $C=4.5$ and $\phi=1 / 3$, this gives $\Lambda=d_{\text {bead }} / 3.76$ and the Berryman relation gives $F=4.5$.

A series of experiments were carried out using fused silica capillaries packed with nonporous, uncoated silica HPLC beads. The capillaries were nominally $10 \mathrm{~cm}$ long, 0.1 and 0.18 $\mathrm{mm}$ inside diameter. The beads were evaporatively packed and the capillary ends thermally fritted to form porous mechanical seals. The formation factor was obtained by measuring the effective conductivity using a phosphate buffer at $\mathrm{pH} 2.7$ (nominally $5 \mathrm{mM} / \mathrm{L}$ ). There was no detectable streaming current hence negligible zeta potential which is consistent with the measurements reported by of Scales [10]: the zeta potential of silica is essentially zero at $\mathrm{pH}$ 2.7. Measurements were performed for several samples of capillaries packed with each bead size, the scatter observed between the different samples was similar to the reproducibility of the individual measurements. Formation factors of 4.7, 4.5, 4.5 and $4.4 \pm 0.15$ (two standard deviations) were measured for packings of $0.6,1.5,3$ and $4.5 \mathrm{~mm}$ beads, respectively. Using 
the Berryman formula these values correspond to porosities of $0.328,0.333,0.333$ and 0.339 , respectively, and a tortuosity near 1.5 for all cases considered

Maximum pressure measurements performed using 1.5, 2.5, 3, 3.5, 4.5 and $5 \mu \mathrm{m}$ beads using both a $5 \mathrm{mM} \mathrm{pH} 7.6$ sodium phosphate buffer and a $5 \mathrm{mM} \mathrm{pH} 9.6$ sodium borate buffer confirm the expected scaling with the inverse of the square of bead diameter. However the pressure per volt applied measured with the phosphate buffer are systematically a factor of 2.5 times greater than that measured with the borate buffer for the same bead size. Measurements made with $0.6 \mu \mathrm{m}$ beads using both these buffers fell well below the value expected from the pore size scaling. The measurements were performed with the downstream electrode (negative terminal) inserted into one leg of an HPLC ' $\mathrm{T}$ ' that coupled the capillary to the pressure transducer. To test the possibility of artifacts due to evolution of the buffer in this small volume select measurements were performed by making the negative terminal connection via a porous glass salt bridge connected to a reservoir of buffer containing the negative electrode. The salt bridge was a nominal $5 \mathrm{~mm}$ long, $0.5 \mathrm{~mm}$ diameter piece of phase separated glass (nominal pore diameter of $5 \mathrm{~nm}$, as custom manufactured by Akagawa Glass, Japan). No significant change in the results were found using the salt bridge.

Electrokinetic Pump Modeling Measurements on the terminal reservoir fluid show that the composition of this fluid evolves over the course of the experiment. However, following the initial transient, pressure and current reach asymptotic valves (i.e. the values stop changing in time). This suggests that the entire system comes into equilibrium. This can occur since the electroosmotic flow continues to transport anode fluid (for a negative pump) to the cathode reservoir while the pressure driven flows transports cathode fluid to the anode reservoir. Simply the electrochemistry drives the anode fluid to lower conductivity and $\mathrm{pH}$ and at the same time the cathode fluid to higher conductivity and $\mathrm{pH}$ while convective stirring (by the electroosmotic- and pressure-driven flows) mixes the fluids thus countering the evolution of the reservoirs. Taking the case of a negative pump and assuming an axial variation in composition along the packed capillary, but taking constant geometrical and formation factors, and applying equation 17 the voltage gradient may be written

$$
\nabla V=\frac{-\Delta V}{L} \frac{1}{\sigma(1-f)(1 / \sigma(1-f))}
$$

Here the over-tilde notation on effective parameters is dropped for notational simplicity. The pressure generated per volt applied is then

$$
\frac{\Delta P}{\Delta V}=\frac{-1}{k} \frac{\langle v / \sigma(1-f)\rangle}{\langle 1 / \sigma(1-f)\rangle}
$$

For a condition of constant electroosmotic mobility, the result of equation $5 \mathrm{c}$ is recovered even for a non-uniform conductivity (i.e. non-constant E-field).

In steady-state, there is no net flow and the net flux of conserved scalars (e.g. Na and atomic boron in a sodium borate buffer) must also be zero. Thus, in steady state, the net flux of fully ionized species (e.g. $\mathrm{Na}$ ) must be zero and thus do not contribute to the current. Rather, for the case of a borate buffer, the current is carried by the negatively charged orthoborates balanced 
by an opposite diffusive/convective flux of neutral orthoborates to achieve a net zero flux of atomic boron.

Species flux relations are applied to the buffer components of the electrolyte, using equilibrium relationships for the buffer chemistry as needed. The concentration of $\mathrm{OH}$ is determined using the equilibrium constant for water and the local concentration of $\mathrm{H}$. The concentration of $\mathrm{H}$ is determined from a condition of local charge neutrality. The pore diameters of the media considered are sufficiently small that the Peclet number (as based on pore diameter) is less than and in some of the cases considered much less than unity. Thus the effect of axial convective stirring is minimal compared to axial diffusion (scaling as the ratio $\mathrm{Pe}^{2} / 48$ ). The species flux equations are thus: purely axial and steady state; are written for a condition of no-net flow; must contain terms for diffusion and E-field driven ion drift (including double layer electromigraiton and electroosmosis), as well as consideration of the streaming convective flux due to the presence of the double layer. Asymptotic solutions suggest that almost the entire capillary is filled with fluid that is essentially the same as the anode (large) reservoir, with a thin diffusion-controlled region at the cathode end where the concentration swings from anode-like to cathode reservoir conditions.

Diffusion alone cannot balance the electromigration over the whole length of the capillary. This is seen by solving the $\mathrm{Na}$ flux relation written for diffusion and electromigraiton only, yielding $\nabla \ln (N a)=-e \nabla V / k T$. Observe that the group $e \Delta V / k T \approx 4000$ for an applied potential of only 100 Volts. This suggests the non-physical condition of a near-infinite ratio of cathode to anode concentrations. This is resolved by including the effect of pressure driven 'streaming-convection' (i.e. the species specific component of the pressure-driven streaming current, see Appendix C equation C1).

This model represents a non-linear system of ordinary differential/algebraic equations with an integral constitutive relation. The problem was simulated using Mathematica 3.0 for a number of the conditions studied experimentally. Several results of the simulations are of note:

1) For the case of a sodium borate buffer at $\mathrm{pH} 9.6$, the ratio of ending to starting current is predicted to be fairly independent of the initial buffer concentrations and bead size. This agrees with the experimental observations for measurements performed using: $2,5,10$, 20,50 and $80 \mathrm{mM}$ initial concentrations with $0.6 \mathrm{~mm}$ diameter beads, and using; 1.5, 2.5, 3.5 and $4.5 \mathrm{~mm}$ beads at an initial concentration of $5 \mathrm{mM}$. For the $0.6 \mathrm{~mm}$ beads the predicted ratio of ending to starting current is nominally $1 / 2$, in good agreement with the experiments. This decrease in current results from a combination of no-net flux of $\mathrm{Na}$, reduced negative ion concentration and a streaming current that opposites the applied current (i.e. the blocked current)

2) A higher performance, of nominally 2 -fold, is predicted for $1.5 \mu \mathrm{M}$ beads using a $5 \mathrm{mM}$ pH 7.6 phosphate buffer compared to a $5 \mathrm{mM}$ pH 9.6 borate buffer. This can be directly attributed to the higher ionic strength of the phosphate buffer (the $\mathrm{Na}$ concentration in the phosphate buffer is nominally $1.5 \times 5 \mathrm{mM} / \mathrm{L}$ ) and to a lesser extent the higher mobility of the phosphate ions that attenuates the value of $\beta$ (compared to borate ions). This is compared to the ionic strength of the borate buffer (the $\mathrm{Na}$ concentration in the borate buffer is nominally $0.66 \times 5 \mathrm{mM} / \mathrm{L}$ ). 
3) For $\mathrm{pH} 9.6$ borate buffer and $0.6 \mathrm{mM}$ beads, the model predicts that the pressure generated per volt applied will increase with increasing concentration from nominally $2 \mathrm{mM}$ to 20 $\mathrm{mM}$ reaching a high concentration value of nominally $8 \mathrm{psi} / \mathrm{volt}$ at a concentration of 25 $\mathrm{mM}$. This prediction is also in good agreement with the measurements. The prediction is that this 'high concentration' value will be maintained to concentrations of $100 \mathrm{mM}$, also in agreement with the experiments. Presumably at even higher concentration the performance will degrade due to suppression of the zeta potential.

To generate a sufficient pressure-driven streaming flux of $\mathrm{Na}$ to balance the flux equations requires an increase in the product $\lambda_{\mathrm{d}}{ }^{2} \zeta$ from that at starting conditions. This can be accomplished through a decrease in $\mathrm{Na}$ and $\mathrm{pH}$ (hence negative ion concentration hence ionic strength). At low initial concentrations (high initial zeta potential, that is near-saturated) the alteration of anode conditions leads to a large increase in $\lambda_{d}{ }^{2}$. Whereas a high initial concentration (low initial zeta potential) the alteration of anode condition produce a substantial increase in zeta potential and a finite increase in $\lambda_{d}{ }^{2}$. In the former case performance is then degraded by substantial double layer overlap, whereas in the later case performance is enhanced.

At high concentrations the model results are in good agreement with the measurements. Whereas a lower concentrations there are systematic deviations, the model predicts lower performance that seen experimentally. This is expected given the assumption of constant mobilities (mobilities are concentration dependent thus the mobilities in the double layer are different that those in the bulk fluid) and the application of a simple double-layer conduction model (the models given above assume a finite thickness double layer, the phenomena involved likely require an accurate model of a partially overlapped double layer). The next step in development of this model is to include corrections for these effects as well as a more sophisticated treatment of the concentration profiles along the length of the capillary. The important result of the model is that the source of a number of what to date have been termed 'experimental artifacts' in EKP testing has been identified. The model represents a powerful tool to develop optimized electrokinetic pump / electrolyte systems.

Conductivity and zeta potential measurements Effective conductivities were measured for several bead-packed capillaries using the $\mathrm{pH} 9.6$ borate, $\mathrm{pH} 7.6$ phosphate and $\mathrm{pH} 2.7$ phosphate buffers as well as a $\mathrm{KCl}$ buffer at $\mathrm{pH}$ 7.5. The measurements were performed for buffer concentrations ranging from 5 to $80 \mathrm{mM} / \mathrm{L}$. The experimental results appear to be consistent with the model described above. The value of the formation factor observed in the high concentration limit is consistent with that measured under a condition of a negligible double layer (i.e. operation at the IEP hence near-zero zeta potential). This result being consistent with the prediction of the model incorporating bulk and double layer conduction. Streaming currents were also measured for the same samples. The results appear to be consistent with the zeta potentials, as a function of buffer concentration, reported by Scales $e t$ $a l$. [10]. For the cases considered the correction for double layer overlap were modest and conditions were well within the limits of the surface conduction model.

Generally streaming current is preferred over streaming potential for measuring zeta potential in porous media. The streaming potential depends on the effective conductivity that includes a complicated dependence on ionic mobilities, double layer structure and micro/macro geometry. Streaming current measurements can be complicated by the effect of electrode 
polarization that is not an issue in streaming potential measurements. In streaming current measurements care must be taken to employ large area or special (e.g. platinized) electrodes to minimize polarization effects.

A series of conductivity, formation factor and zeta potential measurements were performed on samples of porous polymers. In these tests it was essential to condition the samples for up to several days, by electroosmotically flowing buffer, until a steady current was achieved. For some of the samples a steady condition could not be achieved (e.g. following lengthy conditioning, the effective conductivity increased after a period without a potential applied) or switching the sign of the potential did not yield the same current. These effects are attributed to leaching out of unreacted monomers from the polymer samples that alter the apparent conductivity and also may coat the electrodes thus altering the measurement.

Electrokinetic pump frequency response Consider the case where the driving electric field is sinusoidal, of the form $E(t)=E_{o} \sin (\omega t)$. The effect of dielectric enhancement (possibly arising from pore-scale diffusion currents) is ignored. Experimentally dielectric enhancement would be seen as a phase shift between the applied field and the current. A characteristic scale in unsteady boundary layer flows is the viscous skin depth $\delta=\sqrt{\mu / \rho_{f} \omega}$ that arises, for example, in the analysis of Stoke's second problem. Stokes' second problem considers the case of a non-flowing fluid in contact with a wall undergoing a steady transverse oscillation. The viscous skin depth is essentially the $1 / e$ penetration depth of this oscillation into the fluid. The viscous skin depth in water at room temperature is nominally one micron at $120 \mathrm{kHz}$. An applied oscillatory electric field produces an axial oscillation of the double layer that appears as Stoke's oscillating wall. For pore diameters smaller that the viscous skin depth, vorticity can diffuse wall-to-wall in less than one cycle and the pore fluid will tend to move in concert with the double layer, in effect like rigid body.

Consider the case of pure electroosmotic flow (i.e. no externally applied pressure gradient). The Stokes equation is linear and thus the solutions for the velocity and pressure field can be linearly separated into electric and mechanical components, the mechanical components being zero in the absence of an externally applied pressure difference. With the double layer thickness small compared to the viscous skin depth, the Stokes equation can then be recast in boundary layer form. The particular solution will be the same as in steady electroosmotic flow. Adding a homogeneous solution, of the form $\exp (-i x / \delta)$, gives

$$
u_{E}=\left(\frac{\varepsilon \zeta}{\mu}\right)\left(\exp \left(\frac{-i x}{\delta}\right)-\frac{\psi(x)}{\zeta}\right) E_{o} \sin (\omega t)
$$

where $\mathrm{x}$ is a measured normal from the solid surface. For a pore radius less than the viscous skin depth the exponential can be expanded, yielding a phase lag of $\Lambda / 2 \delta$ and a damping of $1 / 2(\Lambda / 2 \delta)^{2}$ to lowest orders.

To test this prediction as section of porous polymer material (nominally $2 \mathrm{~mm}$ long, $1 \mathrm{~mm}$ diameter, mean pore size of $0.5 \mu \mathrm{m}$ ) was installed in a through-hole in a glass plate. Both sides of the porous plug were fitted with electrodes and both sides of the plate were fitted with sealed fluid reservoirs containing a $5 \mathrm{mM}$ pH 7.6 phosphate buffer. Both reservoirs were connected to ambient pressure through nominal $20 \mathrm{~cm}$ long sections of $0.1 \mathrm{~mm}$ ID capillary 
that served to equalize the static pressure while being totally resistant to fluid oscillations. A bubble of air was injected into the lower reservoir. A microphone (B\&K 4138 with 2618 preamplifier) was sealed into the upper reservoir at a high point containing a small bubble of air. The absolute sensitivity of this microphone is $1.07 \mathrm{mV} / \mathrm{Pa}$ and the frequency response is flat to $\pm 1 / 2 \mathrm{db}$ from $10 \mathrm{~Hz}$ to $50 \mathrm{kHz}$ and to within $\pm 1 \mathrm{db}$ up to $140 \mathrm{kHz}$. For small pressure deviations (from ambient) the volumetric flowrate of the pump can be related to the recorded pressure via the perfect gas law (i.e. the peak-to-peak pressure swing is in direct proportion to the peak-to-peak volume displacement). The data were recorded on a Tektronix 2440 digital oscilloscope that was also used for signal averaging as required. At low frequencies an Omega type 237 pressure transducer was employed. Peak-to-peak pressures were recorded as a function of frequency for constant amplitude drive. The ideal result should follow a response that falls inversely with frequency (i.e. for perfect response the volume displacement of an EKP scales linearly with the length of time pumping forward and backward per cycle). Above $1 \mathrm{~Hz}$ the Omega transducer displayed poor frequency response (this device is not intended for $\mathrm{AC}$ operation). However the data below $1 \mathrm{~Hz}$ are consistent with the prediction and these extrapolated to higher frequencies agree well with the microphone data from $10 \mathrm{~Hz}$ to $20 \mathrm{kHz}$. Above $20 \mathrm{kHz}$ the microphone data fall systematically below the perfect response curve. One possible explanation is that at these high frequencies finite size effects become important as artifacts in the measurement (e.g. the acoustic wavelength corresponding to the size of the air bubble, nominally 6 to $8 \mathrm{~mm}$, is $50 \mathrm{kHz}$ in air). To test the high frequency response, the experiment needs to be rerun using helium rather than air. The final result is that from DC to above $20 \mathrm{kHz}$ the measured frequency response of an EKP is in effect ideal and it is possible that this may extend to even higher frequencies.

A second issue that complicates the $\mathrm{AC}$ response of an EKP is one of electrical circuit impedance. Complex impedance measurements $(0.001 \mathrm{~Hz}$ to $1 \mathrm{MHz})$ were made an a $10 \mathrm{~cm}$ section of 100 micron ID capillary packed with 1.5 micron diameter non-porous beads. The results show the system totally dominated by the purely resistive component associated with the pore fluid DC conductivity. This result also confirms the assumption that dielectric relaxation phenomena can be properly neglected. For larger diameter devices, employing larger area electrodes, electrode capacitance may reduce the frequency response of the system.

Nanoporous Matrix Materials Thus far, non-conducting non-porous matrix materials have been considered. However many matrix materials in use are 'nanoporous' (i.e. containing nanometer-scale internal porous structure). For example: porous HPLC packing materials are spherical monodisperse beads with 1 to $20 \mathrm{~nm}$ internal pore structure; or certain porous polymers that have micron-scale porous structure and nanometer-scale internal porous structure. Here, 'nanoporous' is taken to refer to porous structure that is order or smaller than the Debye length. Transport in nanoporous materials is thus purely by diffusive and ionic fluxes. The JKS theory can be recast to consider the case of current transport controlled by surface conduction, introducing a second set of porous material descriptors that are complementary to $F$ and $\Lambda$. However the current state of models does not provide for applying the two limiting cases simultaneously. In effect a new model must be developed to address the added effects of ionic conduction through nanopores in the solid matrix. This new model will need to introduce the complication of applied E-field components directed normal to the microporous surface. 
Several experimental measurements have been performed on nanoporous materials. Conductivity measurements performed on capillaries packed with porous HPLC beads show a 2 to 3 fold increase in current compared to a capillary packed with like-size non-porous beads. However pressure generation measurements with similar sized porous and non-porous beads yield similar results. The evolution of terminal reservoir conductivity is found to be 2 to 3 times faster with porous than with non-porous beads. This is expected since the porous materials is essentially an ion-selective current carrier favoring transport of the double layer forming ions. A series of conductivity, formation factor, zeta potential and pressure generation studies is in progress on samples of porous polymer packed capillaries (both sulfonic acid and quatenary amine modified to provide negative and positive pumps, respectively). The initial results indicate a noticeably higher effective conductivity, again attributed to the nanoporous hence ion-selective nature of these materials.

A tentative description of nanoporous materials can be based, by analogy, to the volumeaveraged flux equation written in terms of apparent transport properties

$$
\begin{gathered}
q=v^{*} \nabla V-k * \nabla P \\
j=-\sigma^{*} \nabla V+v^{*} \nabla P
\end{gathered}
$$

For non-nanoporous materials the apparent properties reduces to the effective properties divided by the formation factor as given above. From the Darcy flowrate measurements on porous and non-porous silica (PS and NPS, respectively) packing of similar sized beads, the values of $\mathrm{k}^{*}{ }_{\mathrm{nps}}$ and $\mathrm{k}_{\mathrm{ps}}$ are found to be essentially equal. Electroosmotic flowrate measurements on the same samples and using a $5 \mathrm{mM} \mathrm{pH} 9.5$ borate buffer indicate that the apparent electroosmotic mobilities for NPS and PS packing of similar sized spheres are also near equal. Electrokinetic pressure generation measurements, again on the same samples, indicate that the ratio $v^{*} / \mathrm{k}^{*}$ are also near equal. Finally conductivity measurements show that the apparent conductivity of PS packing is noticeably larger (by factors of 2 to 4 times) than a packing of like-sized NPS confirming the expected difference between $\sigma^{*}{ }_{p s}$ and $\sigma^{*}{ }_{n p s}$.

To develop a model for conductivity in nanoporous media requires some knowledge of the internal structure of the media. Electron micrographs of nanoporous silica beads are inconclusive regarding the question of the through-connectivity of the particles. Porous silica beads are formed either: as a sil-gel by gelation of soluble silicates or coalescence of fumed silica; or as a xerogel or sol-gel by a sol-aggregation. The sil-gel particle appears to be a cluster of uniform subparticles (typical sil-gel particle properties: 50\% porosity, 1 to $10 \mathrm{~nm}$ internal pores, $150 \mathrm{~m}^{2} / \mathrm{gm} \mathrm{BET}$ area, narrow pore-size distribution, mechanically strong.) The xerogel particle appears sponge-like or like a material formed by spinodal decomposition (typical xerogel particle properties: $70 \%$ porosity, 1 to $10 \mathrm{~nm}$ internal pores, $300 \mathrm{~m}^{2} / \mathrm{gm} \mathrm{BET}$ area, broad pore-size distribution, mechanically weak.) A further class of porous particle is available, termed a 'perfusive' packing, that has a highly bimodal internal pore distribution with larger pores penetrating through the particle and these having smaller side pores. It is also worth note that modern NPS particles are often described as having a solid core and a porous outer layer (e.g. a porous sil-gel or xerogel like coating on a solid core).

In the case that the E-field has a component normal to the microporous surface, the analogy between current and flowrate is lost, and a completely new theory is required. If the E-field components remain parallel to the microporous surface then: If surface conduction remains a 
correction to bulk conduction, the theory given above applies. Whereas, if surface conduction becomes dominant then the JKS theory using the complementary descriptors $F^{*}$ and $\Lambda^{*}$ must be used with the observation that as of yet there is no convenient relationship for $M^{*}$.

Dynamic Length Scale

There is no unique relationship between a given pore size distribution and the dynamical length scale $\Lambda$. The pore size distribution is often determined by mercury $(\mathrm{Hg})$ porosimitry, the basic measurement being the intruded volume of a porous sample as a function of applied $\mathrm{Hg}$ pressure. Typical porosimitry data, in going from low to high $\mathrm{Hg}$ pressures, begins with a response having positive curvature then switching to a response that has negative curvature. The first inflection point is taken to correspond to a percolation threshold at which the $\mathrm{Hg}$ first penetrates completely through the sample. This threshold or 'critical' pressure can be related to a critical diameter $D_{\mathrm{c}}$ via the Washburn or like relation. Katz and Thompson [3] and Banavar and Johnson [11] have considered a possible relation between this critical diameter and the length scale $\Lambda$. Their results suggest the scaling $\Lambda=M^{-1 / 2} D_{C}$. Taking $M=1 / 32$, their data analysis suggests a value of $\Lambda$ between $1 / 3$ and $1 / 5 D_{\mathrm{c}}$. It is noteworthy that the measured value of $D_{\mathrm{c}}$ for a bead-packed medium at a porosity of $1 / 3$ is near $d_{\text {bead }} / 3.8$, is consistent with this range and corresponds well to the estimate given above, $\Lambda=d_{\text {bead }} / 3.76$, as based on the Kozeny-Carman relation and Berryman's formula for the formation factor.

Saeger et al. [5] have reported a numerical analysis of simple, face-centered and bodycentered cubic (SC, FCC and BCC, respectively) lattices of monodisperse spheres covering the bead- to bubble-pack limits. Their results confirm the value $M \approx 1 / 32$ with the exception of SC arrays in the bubble pack limit. In the SC bubble-pack limit, they find that $M$ will tend towards larger values as the size of the interconnecting pores (orifices in the bubble pack limit) become small compared to the unit cell size. They do not find this behavior for FCC and $\mathrm{BCC}$ arrays in the bubble pack limit. The deviation in the SC case is attributed to a fundamental geometrical difference: in the bubble-pack limit, the $\mathrm{SC}$ array displays a monodisperse throat pore size distribution whereas the connections between unit cells in the $\mathrm{BCC}$ and FCC arrays are bi-continuous. This suggests that foams or sol-gel derived structures (i.e. bubble-pack limit) should exhibit $M \approx 1 / 32$ except for the pathological but physically unlikely case of a mono-continuous SC-array in the limit of vanishing interconnecting orifice diameter.

The preferred method of determining the length scale $\Lambda$ is to measure the pressure-driven flowrate, $Q$, through a sample of know length and cross sectional area. For operation with a finite conductivity electrolyte under short circuit conditions or operation at a $\mathrm{pH}$ corresponding to the matrix material IEP, the pressure-driven flowrate is given by $Q=-M \Lambda^{2} \triangle P A / \mu F L$. Obviously the formation factor is required to interpret this measurement that then provides a value for $\Lambda$ under the assumption that $M \approx 1 / 32$. The most accurate means of measuring low flowrates is to use apply Darcy's law to the pressure drop measured across a known sample (e.g. a length of open capillary) as a flow meter. Alternatively the transit time of an injected fluorescent marker can be used. A flowratecontrolled HPLC pump can be used to provide pressure and meter flow. However an absolute flowrate is required and it is very difficult to accurately produce the extremely low flowrates required for these measurements using this type of pump (this is a well known problem in using these pumps for capillary, termed 'nanobore' HPLC applications). Thus it is essential to 
regularly and meticulously calibrate the pump at the running pressures, using a method similar to the direct flowrate measurement described here, to achieve accurate measurements of $\Lambda$.

Application of porous materials in microfluidic systems It is useful to consider the potential applications of porous materials in microfluidic system, as delimited by zeta potential (as a surface parameter), the formation factor (as a structural or geometric parameter) and the dynamic scale $\Lambda$ (as a physical length scale). It is assumed that the pore geometry number is essentially constant (order 1/32). The object is to identify particular regions in the parameter three-space (e.g. $\zeta, F$ and $\Lambda$ space) that correspond to different microfluidic devices. Such devices include:

Electokinetic pumps: The objective is high efficiency generation of pressure and flowrate. Preferably high zeta potential (greater than $70 \mathrm{mV}$, of both positive and negative type), low formation factor, mechanically strong, pore size down to $50 \mathrm{~nm}$ for high pressure and up to $500 \mathrm{~nm}$ for high flowrate variants. Narrow pore-size distribution. Not nanoporous.

Hydraulic flow restrictors: The objective is an element that is non-ion selective and essentially transparent to electroosmotic flow but restricting pressure-driven flow. Pore size of order 500 $\mathrm{nm}$, adjustable zeta potential (to match that of the remaining flow system), low formation factor. Definitely not nanoporous.

Salt bridges: The objective is an element that restricts both pressure and electroosmotic-driven flows but provides for a non-ion selective current flux. Pore size of order 10 to $50 \mathrm{~nm}$ and low zeta potential (less than $5 \mathrm{mV}$ but sufficient to promote wetting of the material), low formation factor, mechanically strong.

Ion-permeable membranes: The objective is an element that restricts both pressure and electroosmotically-driven flows but provides for an ion selective current flux. Pore size of order $5 \mathrm{~nm}$, moderate zeta potential (both positive and negative), low formation factor. Preferably not nanoporous.

Separation column (for electro-chromatography): Pore sizes from 200 to $1500 \mathrm{~nm}$, variable zeta potential (both positive and negative), low formation factor, variable surface chemistry. Preferably with narrow pore-size distribution and preferably not nanoporous.

Separation column (for HPLC): Pore sizes from 500 to $1500 \mathrm{~nm}$, variable zeta potential (positive, negative and near negligible), low formation factor, mechanically strong, variable surface chemistry. Both nanoporous (preferably with narrow bimodal pore-size distributions) and non-nanoporous (preferably with a narrow pore-size distribution).

In considering these various devices it becomes obvious that a relatively narrow pore-size distribution is generally preferred. It also becomes obvious that a low formation factor is in general preferable. The formation factor must be greater than the inverse of the porosity, making minimum practical values of order 2 to 3 . Whereas formation factors much greater than 5 (say) are not desirable. Thus devices can be delimited in the two-space of zeta potential and dynamic pore scale. The domains corresponding to the set of devices described above are shown sketched in figure 1. Further non-nanoporous materials are preferred and sometimes mandatory with the exception of certain types of pressure-driven separation columns (for sizeexclusion, say). Nanoporous materials are strongly ion-selective and draw excess current 
which are both detrimental in electro-chromatographic as well as in EKP applications. Wallenberg and Paul [12] have shown how the effects of ion selectivity degrade the performance on micellar separations in open channels as well as when using double reservoirs coupled via both $5 \mathrm{~nm}$ pore glass and nanoporous polymer salt bridges. Garguilo et al. [13] have shown how the ion selectivity of nanoporous separation columns induces pressuredriven stirring at the end of the column thus degrading the ability to detect a sharp band as it elutes. A further case of complications introduced by ion-selectivity is discussed in Appendix C. Finally, the lack of a suitable theory to describe current and flow in nanoporous materials makes it difficult to develop engineering design rules for use of these materials.

Conclusion Formal volume averaging of the governing equations for flow and current transport in porous media has been applied to yield a set of one-dimensional axial flux equations. These relations form the basis for analysis of a wide range of microflow geometries, specifically flow in porous media and in complex microchannel systems. The relations are written in terms of axial gradients in the driving fields (e.g. applied electrical potential and pressure differences) and include the effect of non-uniform transport properties (e.g. porous permeability, electrical conductivity and surface zeta potential). A method for analyzing systems of porous elements (analogous to that used for the analysis of an electrical resistor network) was presented. Using the flux relations, the eight classes of current-flow interactions in a porous media were identified and relations for these process were given for the case of non-uniform transport properties.

A new set of porous media descriptors were presented, specifically: the formation factor, $F$, that represents the geometry of the system; the zeta potential, $\zeta$, as a surface parameter, and; a dynamic length scale, $\Lambda$. These three parameters completely replace the traditional set of essentially empirical parameters (e.g. hydraulic formation factor or tortuosity) when combined with the experiential and theoretical observation that the pore geometry number, $\mathrm{M}$, is essentially a constant. This leads to a relationship for the Darcy permeability of the form $\mathrm{k}_{\mathrm{D}}$ $=M \Lambda^{2} / F$. Methods of measuring these parameters were described.

The frequency response of an electrokinetic pump was analyzed. Theoretically, the response of flow in an EKP to a change in driving voltage should be 'ideal' (i.e. a fluid response near rigid-body motion) for driving frequencies up to order $100 \mathrm{kHz}$. Experimental results confirm this prediction within the capabilities and resolution of the instrumentation applied.

Axial electrochemically-generated non-uniformity in fluid composition, as an artifact of the testing methods used to date, was found to be a significant effect. Consideration of the processes involved provides a means of reconciling all of the available electrokinetic pump data. Further, these considerations suggest methods of improving electrokinetic pump operation (particularly at high pressures) and efficiency. 


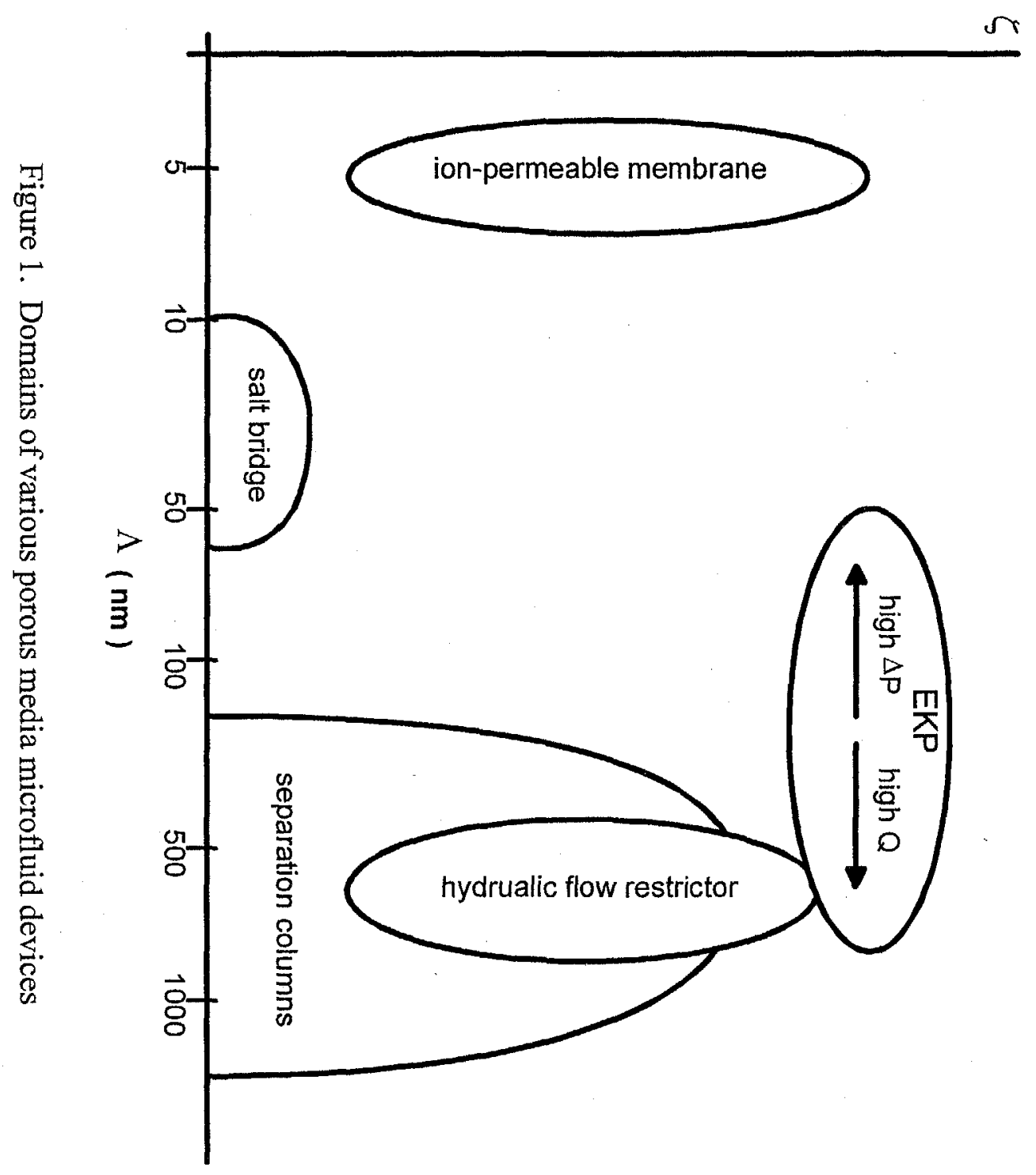




\section{References}

1. P. H. Paul, 'Microfluidic Engineering,' (Sandia National Laboratories, 1999), report SAND99-8212.

2. D.L. Johnson, J. Koplick and R. Dashen, J. Fluid Mech. 176, 379-392 (1987).

3. A. J. Katz and A. H. Thompson, Phys. Rev. B 34, 8179-8181 (1986).

4. D. L. Johnson, J. Koplik and L. M. Schwartz, Phys. Rev. Let. 57, 2564-2567 (1986)]

5. R. B. Saeger, L. E. Scriven and H. T. Davis, J. Fluid Mech. 299, 1-13 (1993).

6. M. Avellaneda and S. Torquato, Phys. Fluids A 3, 2529-2540 (1991).

7. A. Revil and P. W. Glover, Phys. Rev. B 55, 1757-1773 (1997).

8. E. B. Cummings, S. K. Griffths, R. H. Nilson and P. H. Paul, 'Conditions for similitude between fluid velocity and electric field in electroosmotic flow,' (Sandia National Laboratories, 1999), report SAND99-8246.

9. J. G. Berryman, Phys. Rev. B 27, 7789-7792 (1983).

10. P. J. Scales, F. Grieser, T. W. Healy, L. R. White and D. Y. C. Chan, Langmuir 8, 965974 (1992).

11. J. R. Banavar and D. L. Johnson, Phys. Rev. B 35, 7283-7286 (1987)

12. S. R. Wallenberg and P. H. Paul, 'Electrokinetically driven separations on microchips: the use of separate high voltage electrode reservoirs and porous bridges to minimize buffer depletion,' (to be submitted to J. Chromo A, 2000)

13. M. G. Garguilo, P. H. Paul and D. J. Rakestraw, 'Fluorescence imaging of pressure and electroosmotically driven flows through packed beds used in micro-high performance liquid chromatography and capillary electrochromatography,' (to be submitted to Anal. Chem., 2000) 


\section{Appendix A. Data useful in modeling electrokinetic flows}

Zeta potential $(\mathrm{mV})$ of silica at $293 \mathrm{~K}$ with a $\mathrm{KCl}$ electrolyte

\begin{tabular}{|c|c|c|c|c|c|c|}
\hline & $\mathrm{pH} 5$ & $\mathrm{pH6}$ & PH7 & $\mathrm{pH} 8$ & $\mathrm{pH9}$ & $\mathrm{pH} 0$ \\
\hline $10^{-1} \mathrm{M}$ & 16.8 & 22.8 & 30 & 34.8 & 37.2 & 38.4 \\
\hline $10^{-2} \mathrm{M}$ & 32.4 & 44.4 & 60 & 68.4 & 72 & 73.2 \\
\hline $10^{-3} \mathrm{M}$ & 50.4 & 68.4 & 90 & 99.6 & 99.6 & 99.6 \\
\hline $10^{-4} \mathrm{M}$ & 66 & 90 & 109.2 & 115.2 & 115.2 & 115.2 \\
\hline
\end{tabular}

Taken from: Scales et al. Langmuir 8, 965-974 (1992)

$$
\begin{gathered}
\text { Dynamic viscosity of water } \\
\mu=2.35 \mathrm{E}-5 \exp (1.77 \mathrm{E} 3 / \mathrm{T}[\mathrm{K}]) \text { poise }
\end{gathered}
$$

Taken from: E. N. Andrade and C. Dodd, Proc. Royal Soc. A 204, 449-464 (1951).

Relative permittivity of water

$$
\varepsilon=87.740-0.4008 \mathrm{~T}+9.398 \mathrm{E}-4 \mathrm{~T}^{2}+1.410 \mathrm{E}-6 \mathrm{~T}^{3} \quad \mathrm{~T} \text { in degrees } \mathrm{C}
$$

Taken from: C. G. Malmberg and A. A. Maryott, J. Res. Nat. Bur. Stand. 56, 1-8 (1956)

pKa for phosphates $\mathrm{PO}_{4}: \quad 2.12,7.21,12.67$

$$
d \mathrm{pK} / d \mathrm{~T}: \quad 0.0044,-0.0028,-0.026
$$

pKa for orthoborates: $\quad 9.21,12.74,13.80 \quad d\left(\mathrm{pK}_{1}\right) / d \mathrm{~T}=-0.008$

pKa for tetraborates: $\quad 4.00,6.54,9.00$

equilibrium constant for neutral ortho and tetra borates: $\log \left\{\left[\mathrm{H}_{2} \mathrm{~B}_{4} \mathrm{O}_{7}\right] /\left[\mathrm{H}_{3} \mathrm{BO}_{3}\right]^{4}\right\}=-2.55$

pKa for TRIS: $\quad 8.075 \quad d(\mathrm{pKa}) / d \mathrm{~T}=-0.028$ 


\section{Appendix B. Assumption of constant permittivity and viscosity}

The charge separation in the double layer causes polarization of solvent molecules near the solid surfaces. Booth [F. Booth, JCP 19, 391-394 (1951)] has considered the effect of dielectric saturation in water and gives a model for the permittivity of the form

$$
\varepsilon(z)=\varepsilon_{R}\left(1-\theta_{e}|\nabla \psi|^{2}\right)
$$

where $\varepsilon_{\mathrm{R}}$ is the DC permittivity, $\theta_{\mathrm{e}}$ for water is $4 \mathrm{E}-18 \mathrm{~m}^{2} / \mathrm{V}^{2}$ at $25 \mathrm{C}$, and $\psi$ is the potential field in the double layer. Substituting this relation into the Possion-Boltzman equation (i.e. $\nabla \bullet \varepsilon \nabla \psi=2 n e \sinh (e \psi / k T)$ for a univalent electrolyte) for a plane-parallel geometry yields

$$
\left(1-B X^{2}\right) X d X=\sinh (y) d y
$$

Here $X=d y / d r, r=x / \lambda_{d}$ with $\lambda_{d}$ the Debye length and $x$ a normal position to the surface, $\mathrm{y}=\mathrm{e} \psi / \mathrm{kT}$, and $\mathrm{B}=6 \theta_{\mathrm{e}} \mathrm{nkT} / \varepsilon_{\mathrm{R}}$. The relation can be integrated to yield a quadratic in $\mathrm{X}^{2}$ that reduces to the same result that would be obtained under the assumption of constant permittivity in the limit that $4 B(\cosh (e \zeta / k T)-1)<<1$. Using experimental values for the zeta potential of silica in water, this limit is found to be well-satisfied for electrolyte concentrations less than $0.1 \mathrm{M}$ (at $0.1 \mathrm{~mol} / \mathrm{L}$ the value is $4.16 \%$ falling to $0.192 \%$ at $10^{-5}$ $\mathrm{mol} / \mathrm{L})$.

Lyklema and Overbeek [J. Lyklema and J. T. G. Overbeek, J. Colloid Sci 16, 501-512 (1961)] have suggested that the field dependence of dynamic viscosity (termed the viscoelectric effect) follows a relation similar to that given by Booth for dielectric saturation. Based on experimental results, Hunter [R.J. Hunter, Colloid Interface Sci. 22, 231-239 (1966)] suggested that the corresponding viscoelectric coefficient is similar in magnitude and opposite in sign to the dielectric saturation coefficient. Using a constant permittivity, the variation in the Stokes equation introduced by the viscoelectric effect is found to be less than $0.7 \%$ at $0.1 \mathrm{~mol} / \mathrm{L}$, falling to less than $0.03 \%$ at $10^{-5} \mathrm{~mol} / \mathrm{L}$.

It is thus reasonable, for the conditions of practical interest in microflow systems, to neglect both dielectric saturation and viscoelectric phenomena in the double layer, hence assume constant viscosity and permittivity. 


\section{Appendix C. Flow in a microchannel partially packed with porous material}

Consider the case of a microchannel of cross-sectional area $A$ and length $L$ a portion of which, $L^{\prime}$, is filled with a porous material the remain length being open. The microchannel is connected between two terminal reservoirs as connected to ambient pressure and containing electrodes with a potential $\Delta \mathrm{V}$ is applied between the electrodes. The capillary and porous packing are taken to be negative zeta potential materials. The electrolyte is taken as aqueous with a common buffer (e.g. TRIS-HCl or Na-borate or Na-phosphate). Two cases are considered: first, the porous-packed end of the microchannel is placed in anode reservoir thus the open or un-packed end is the cathode reservoir, and second; vis versa. Assuming a finite zeta potential, when the packing is nanoporous or when the ratio $\lambda_{d} / \Lambda$ in the packing is order 0.02 or greater then the current in the packing is carried more by the counterions (i.e. for a negative zeta potential material, the positive ions in the electrolyte) than by the coions. Whereas in the open section the current is essentially carried equally by both ions. This is shown by writing the current flux for the $k^{\prime}$ th species

$$
j_{k}=-\left(e z_{k}\right)^{2} b_{k} N_{k}\left[1+\frac{4 \lambda}{\Lambda} 2\left(1+\frac{2 \varepsilon k T}{\left(e z_{k}\right)^{2} \eta b_{k}}\right)\left(\exp \left(\frac{-y_{k}}{2}\right)-1\right)\right] \frac{\nabla V}{F}+e z_{k} N_{k} q
$$

In the brackets, the first term corresponds to simple ionic conduction, the following two terms are the electromigration and electroosmotic surface contributions (the later order $1 / 2$ the former). The final term on the right-hand of equation $\mathrm{C} 1$ reflects purely convective transport. Summing over all species would yield the result of equation 15 above. In summing equation $\mathrm{C} 1$ over all species the convective term vanishes owing to the condition of charge neutrality. For negative or positive solid materials, the counter-ion (i.e. the double layer forming ion) is positively or negatively charged. For the counter- and co-ions, $y_{k}=e z_{k} \zeta / \varepsilon k T$ is negative and positive, respectively. Thus the surface conductance enhances counter- and degrades coion fluxes in the porous material as compared to that in the open section (where double layer effects are completely negligible and current is carried by simple ionic conduction).

The total current must be conserved in any cross section. Thus for the case where the porous material is in intimate contact with the anode (source) reservoir: The excess flux of positive ions through the packing will create an increase in positive ion concentration at the downstream end of the packing (due to the flux imbalance, negative ion concnetrations will also increase at this interface). This will increase the ionic strength hence conductivity and $\mathrm{pH}$ at the interface, decreasing the zeta potential and creating an electrosmotically induced pressure gradient (owing to the axial non-uniformity in zeta potential). There will be an increase in pressure at the interface, required to conserve flowrate. This increase in pressure stirs the fluid just down-stream of the interface. This convective stirring would degrade the peak-height of a chromatographic band eluting from the packing into the open section. It is preferable to optically detect just down-stream of the packing, to mitigate scattering and background fluorescence or absorption due to the packing. This will be at the cost of some degradation in performance, due to stirring-driven band broadening in the detection region, when ion-selective packing materials are used in electro-chromatography,

For the case where the open end is placed in the anode reservoir: The flux imbalance will lead to a decrease in positive and negative ion concentration at the interface (now inlet to the 
porous material). This will decrease the local $\mathrm{pH}$ leading to a drop in zeta potential at the inlet of the porous section. To conserve flowrate this will drive a decrease in pressure at the interface that can eventually lead to boiling of the fluid and an abrupt cessation of current. Both of the cases described here have been observed and documented experimentally.

Consider then a microfluid system designed for chemical analysis using electrochromatography in porous media packed columns. Present designs would have the column inlet just downstream of an open channel injector. The issues discussed above suggest that even moderately ion-selective porous materials cannot be disposed in microchannel systems unless the fluid source end of the material is in contact with the source reservoir. In effect ionselective packings (e.g. porous HPLC beads or nano-porous polymers) cannot be used for electro-chromatography as performed using current chip architectures. 


\section{DISTRIBUTION:}

$1 \quad 1$ MS0188 $\quad$ D. Chavez, 4532 for LDRD Office

25 MS9051 P. H. Paul, 8351

3 MS9018 Central Technical Files, 8940-2

1 MS0899 Technical Library, 4916

1 MS9021 Technical Communications Dept, 8815/

Technical Library, MS0899, 4916

1 MS9021 Technical Communications Dept, 8815. For DOE/OSTI

1 MS0161 Patent and Licensing Office, 1150 